\title{
Case Series- Heterogeneity of Primary Myelofibrosis- A Challenge to the Clinician
}

\section{Gunawardena D* and Bavanthan J}

Department of Pathology, Faculty of Medical Sciences, University of Sri Jayawardenapura, Sri Lanka

\section{Introduction}

Primary myelofibrosis (PMF) is a myeloproliferative neoplasm characterized by stem cell derived clonal myeloproliferation and extramedullary haematopoiesis (EMH). Myelofibrosis can present as a de novo disorder or evolve secondary to previous polycythemia vera or essential thrombocythemia (post PVMF or post ETMF) [1]. Primary myelofibrosis (PMF) is the least frequent among the chronic myeloproliferative diseases. One study reported an estimated incidence of 1.5 per 100,000 per year 2 PMF occurs mainly in the middle aged and elderly population. The median age at presentation is 67 years [2]. The primary pathogenic mechanism is a clonal stem-cell disorder that leads to ineffective erythropoiesis, dysplastic-megakaryocytic hyperplasia, and an increase in neutrophils with predominance in immature forms $[3,4]$.

The disease is clinically characterized by progressive anemia, constitutional symptoms and splenomegaly. The laboratory findings show a leuko-erythroblastic blood film, tear drop poikilocytosis, reticulin fibrosis in bone marrow and elevated levels of various inflammatory and pro-angiogenic cytokines [1].

The majority (approximately 45-68\%) of the patients harbor JAK2V617F mutation [5]. MPLW515L mutations were described in JAK2V617F mutation-negative PMF. Recent discovery of the CALReticulin mutation has improved the genetic detection of MF to about $80 \%$ [6]. PMF has poor prognosis [7-11]. Causes of death are infections, hemorrhage, thrombosis or progression to leukaemia.

Herein we present the clinico-hematological profile of three cases fitting the diagnosis of PMF and one case with post PVMF. These four cases show the diversity of the disease with regard to laboratory, clinical and prognostic features.

\section{Case 1}

This 58 year old male patient presented with fever, loss of appetite and weight loss of two weeks duration. On examination, he had mild pallor and moderate splenomegaly. There was no lymphadenopathy, hepatomegaly or bleeding manifestations. Full blood count revealed haemoglobin-10.2 g/dL, total leukocyte count $29.7 \times 103 / \mu \mathrm{L}$ and platelet count $229 \times 103 / \mu \mathrm{L}$. Peripheral blood smear showed normochromic normocytic red cells with no tear drop cells or nucleated red cells. White cells showed leukocytosis with complete spectrum of neutrophil lineage including myoblasts. Platelets were normal. The bone marrow trephine biopsy showed marked fibroblastic proliferation with clustering and dysplasia of megakaryocytes (Figure 1). Other cell lines were markedly suppressed. Reticulum was grade [3]. BCR - ABL was negative. JAK2V617F was positive. The patient was treated with hydroxycarbamide/hydroxyurea 500mg daily. At three months, his blood counts are stable and spleen size was regressed by $3 \mathrm{~cm}$.

\section{Case 2}

This 67 year old female presented with loss of appetite and weight loss of six months duration. At presentation she was mildly pale and had hepatosplenomegaly, no lymphadenopathy or bleeding manifestations. Full blood count showed hemoglobin $10.3 \mathrm{~g} / \mathrm{dl}$, total leukocyte count $27.6 \times 103 / \mu \mathrm{L}$ and platelet count 286,000 . Peripheral blood smear revealed normochromic normocytic red cells, tear drop cells and nucleated red cells. White cells showed leukocytosis with complete spectrum of granulocytic lineage including myeloblasts (1\%). Platelets were normal. Bone marrow trephine biopsy showed marked fibroblastic proliferation (Figure 2), lack of other hematopoietic elements with a streaming pattern in the intertrabecular area. Reticulin was grade 4. BCR - ABL was negative. This patient was treated with hydroxycarbamide/hydroxyurea $500 \mathrm{mg}$ daily. At two months of follow up, blood counts were normal, but her spleen was palpable at 18 $\mathrm{cm}$ below the costal margin.

\section{Case 3}

This 69 year old male presented with dyspnea and loss of appetite of $2 / 52$ duration. On examination he had pallor but no organomegaly or lymphadenopathy or bleeding manifestations. Full blood count revealed hemoglobin, $4.9 \mathrm{~g} / \mathrm{dl}$, total leukocyte count $4.64 \times 103 / \mu \mathrm{L}$ and a platelet count 58,000 . Peripheral blood film revealed normochromic, normocytic red cells, macrocytes, no tear drop cells or nucleated RBCs. There were no abnormal white blood cells. Platelets were low. The

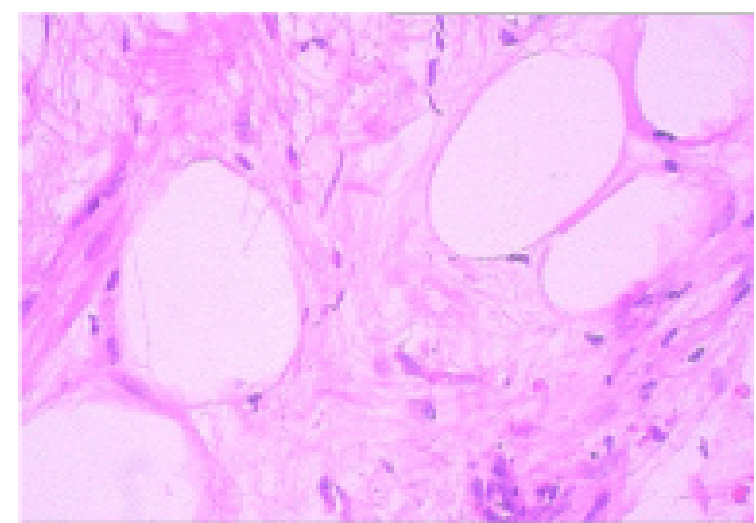

Figure 1: Bone marrow trephine biopsy fibroblastic proliferation with clustering and dysplasia of megakaryocytes Case 1(H and E stain 40X).

*Corresponding author: Dr. Dammika Gunawardena, Department of Pathology, Faculty of Medical Sciences, University of Sri Jayawardenapura, Sri Lanka, Tel: 94 11 2758718; E-mail: dammika.g@hotmail.com

Received April 29, 2015; Accepted May 01, 2015; Published May 05, 2015

Citation: Gunawardena D, Bavanthan J (2015) Case Series- Heterogeneity of Primary Myelofibrosis- A Challenge to the Clinician. J Blood Lymph 5: 138. doi:10.4172/2165-7831.1000138

Copyright: (c) 2015 Gunawardena D, et al. This is an open-access article distributed under the terms of the Creative Commons Attribution License, which permits unrestricted use, distribution, and reproduction in any medium, provided the original author and source are credited. 
Citation: Gunawardena D, Bavanthan J (2015) Case Series- Heterogeneity of Primary Myelofibrosis- A Challenge to the Clinician. J Blood Lymph 5: 138. doi:10.4172/2165-7831.1000138

bone marrow trephine biopsy showed dense fibroblastic proliferation (Figure 3) with megakaryocytic clustering and dysplasia resembling idiopathic myelofibrosis. Reticulin was grade 3 . This patient was treated with Erythropoietin 10,000 IU weekly for 4 months with no significant response. He was then treated with thalidomide and oral prednisolone, for which he developed disturbing side effects of severe constipation and peripheral neuropathy with no improvement in the blood counts, which warranted us to stop the drug. Finally he was started on Danazol $300 \mathrm{mg}$ daily which we increased to $500 \mathrm{mg}$ later. At 3 months of follow up his hemoglobin was $6.0 \mathrm{~g} / \mathrm{dl}$. Results are yet to be shown and still he is on regular blood transfusions once in three weeks.

\section{Case 4}

This 55 year old diagnosed patient with JAK 2 positive primary proliferative polycythemia was well controlled on hydroxyurea $500 \mathrm{mg}$ daily and aspirin for 6 years. She eventually showed a trilineage increment of blood counts which needed to be controlled with a higher dose of hydroxycarbamide/hydroxyurea. Her blood counts were well controlled with dose adjustments with hydroxyurea for the following 6 years and then, she developed a leukoerythoblstic blood picture with tear drop poikilocytes. Her counts were WBC -64,300/ $\mu \mathrm{L}, \mathrm{Hb}-11.0 \mathrm{~g} / \mathrm{dL}$, Platelets - $1485,000 / \mathrm{mm}^{3}$. White blood cells showed marked leukocytosis with complete spectrum of neutrophil lineage including myeloblasts. The bone marrow Trephine biopsy was markedly hypercellular with fibroblastic proliferation, megakaryocytic proliferation with dysplastic megakaryocytes, markedly suppressed

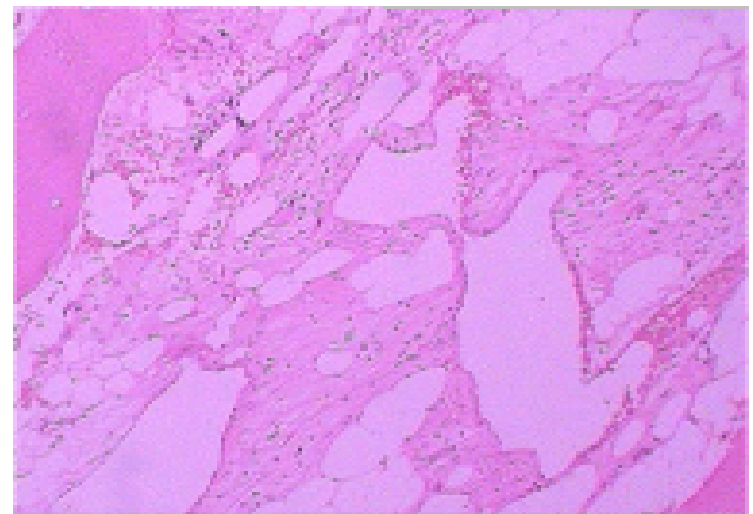

Figure 2: Bone marrow trephine biopsy. Case-2 ( $\mathrm{H}$ and $\mathrm{E}$ stain 40X).

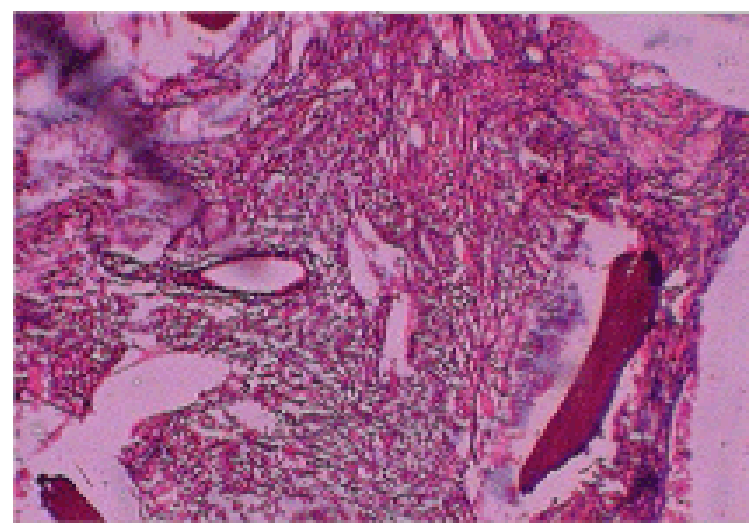

Figure 3: Bone marrow trephine biopsy showed dense fibroblastic proliferation. Case 3-(Reticulin stain $40 \mathrm{X}$ ). other haemopoietic elements, vascular sinusoidal proliferation and reticulin stain was grade 3 . The patient continued with dose adjustment of hydroxycarbamide. At one year of follow up she maintained hemoglobin between 9-10 g/dL with platelet counts ranging from $400-800 \times 109$ and the white cell count was controlled around 15,000 $\times 109 / \mathrm{L}$. She now has palpable splenomegaly. Abdomina untrasound shows a splenomegaly $14 \mathrm{~cm}$ below costal margin. She fulfilled all the diagnostic criteria of MF secondary to PV.

\section{Discussion}

Myeloproliferative neoplasms are a collection of diseases that have overlapping clinico-pathological features and this is a challenge to the hematologist distinguishing one from the other. This is particularly true in ET and early phase/pre-fibrotic form of MF.

The diagnosis of primary myelofibrosis is based on the 2008 World Health Organization (WHO) criteria, which include histopathological, morphological, clinical, and molecular-cytogenetic variables. To confirm a diagnosis of PMF, patients must meet all three major criteria plus two minor criteria. The major criteria are largely histopathologybased. For example, the presence of increased megakaryopoiesis with a preponderance of atypical megakaryocytes is an important feature of PMF. Such findings are usually associated with increased bone marrow cellularity. The later, although less frequently, can also occur in cases of ET. The second major criterion is excluding the other MPD (CML,MDS or PV) which have a similar presentation. The third criterion is demonstration of JAK2V617F or the absence of reactive marrow fibrosis [3]. Minor criteria include the presence of a leukoerythroblastic blood picture, a high Lactate Dehydrogenase (LDH) level, splenomegaly, and/or anaemia.

First two patients have massive splenomegaly, leukoerythroblastic blood picture with tear drop poikilocytosis but no cytopenias. In addition to bone marrow fibrosis, megakaryocytic dysplasia was present with clustering of megakaryocytes. BCR-ABL was negative in both cases and JAK2V617F mutation was positive in the first case. They were diagnosed as fibrotic stage of primary myelofibrosis and therefore they have been treated with hydroxycarbamide $500 \mathrm{mg}$ daily. The extra medullary hematopoiesis was well controlled with improvement of their splenomegaly.

In case 3 , compared to previous cases, patient had no organomegaly, leukoerythroblastic blood film or tear drop poikilocytosis but had pancytopenia. Bone marrow histology showed features resembling idiopathic bone marrow fibrosis such as clustered megakaryocytes and dense fibrosis. Currently patient is on regular blood transfusions.

In case 4, patient had a previous history of PV with grade 3 bone marrow reticulin fibrosis and increasing splenomegaly. Patient was treated with hydroxycarbamide/hydroxyurea. The International Prognostic Scoring System (IPSS) 8 uses the following 5 risk factors for estimating survival at the time of diagnosis: age $>65$ years, hemoglobin level $<10 \mathrm{~g} / \mathrm{dL}$, leukocyte count $>25 \times 109 / \mathrm{L}$, circulating blasts $\geq 1 \%$, and the presence of constitutional symptoms. The presence of 0,1 , 2 , and $\geq 3$ adverse factors define low, intermediate 1 , intermediate 2 , and high-risk disease with median survivals of $11.3,7.9,4$, and 2.3 years, respectively. Later IPSS was modified to Dynamic IPSS (DIPSS). Most recently, DIPSS9,10 was upgraded to DIPSS-plus11 by the incorporation of 3 additional IPSS/DIPSS independent risk factors including red cell transfusion need, platelet count $<100 \times 109 / \mathrm{L}$, and unfavorable karyotype; the latter includes sole complex karyotype or 2 abnormalities that include $+8,-7 / 7 q-$, I (17q), inv (3), $-5 / 5 q-$, 
Citation: Gunawardena D, Bavanthan J (2015) Case Series- Heterogeneity of Primary Myelofibrosis- A Challenge to the Clinician. J Blood Lymph 5: 138. doi:10.4172/2165-7831.1000138

Page 3 of 3

$12 \mathrm{p}-$, or 11q23 rearrangement 3. The eight (8) DIPSS-plus risk factors are used to define low (no risk factors), intermediate 1 (1 risk factor), intermediate 2 ( 2 or 3 risk factors), and high ( $\geq 4$ risk factors) risk groups with respective median survivals of $15.4,6.5,2.9$, and 1.3 years.

All the cases above come under the high risk category. These cases illustrate the significant heterogeneity that is seen in patients with MF which could cause a challenge in the diagnosis and management of myelofibrosis.

\section{References}

1. Reilly JT, McMullin MF, Beer PA (2010) Guideline for the management and diagnosis of myelofibrosis. Br J Haematol 158: 431-560.

2. Tefferi A (2014) Pathogenetic mechanism in primary myelofibrosis. Clinical manifestations and diagnosis of primary myelofibrosis.

3. Cervantes F, Dupriez B, Passamonti F, Vannucchi AM, Morra E, et al. (2012) Improving Survival trends in primary myelofibrosis. J Clin Oncol 30: 29812987.

4. Passamonti F, Cervantes F, Vannucchi AM, Morra E, Rumi E, et al.(2010) A dynamic prognostic model to predict survival in primary myelofibrosis:a study by the IWGMRT (International Working Group for Myeloproliferative Neoplasms Research and Treatment) Blood. 115: 1703-1708.
5. Tefferi A (2011) How I treat myelofibrosis, Blood:117

6. Vannucchi AM, Rotunno G, Bartalucci N,et al. (2014) Chromic Myeloproliferative Neoplasias:Calreticulin mutation-specific immunostaining in myeloproliferative neoplasms:Pathogenic insight and diagnostic value.Leukemia 28: 18111818.

7. Vardiman JW, Thiele J, Arber DA, Brunning RD, Borowitz MJ, et al. (2009) The 2008 revision of the World Health Organization (WHO) classification of myeloid neoplasms and acute leukemia:rationale and important changes. Blood 114 937-951.

8. Cervantes F, Dupriez B, Pereira A, Passamonti F, Reilly JT et al. (2009) New prognostic scoring system for primary myelofibrosis based on a study of the International Working Group for Myelofibrosis Research and Treatment. Blood 113: 2895-2901.

9. Passamonti F, Cervantes F, Vannucchi AM, Morra E, Rumi E, et al. (2010) Dynamic International Prognostic Scoring System (DIPSS) predicts progression to acute myeloid leukemia in primary myelofibrosis. Blood 116: 2857-2858.

10. Passamonti F, Cervantes F, Vannucchi AM, Morra E, Rumi E, et al. (2010) A dynamic prognostic model to predict survival in primary myelofibrosis:a study by the IWG-MRT (International Working Group for Myeloproliferative Neoplasms Research and Treatment) Blood 115: 1703-1708.

11. Gangat N, Caramazza D, Vaidya R, George G, Begna K, et al. (2011) DIPSS plus: a refined Dynamic International Prognostic Scoring System for primary myelofibrosis that incorporates prognostic information from karyotype, platelet count, and transfusion status. J Clin Oncol 29: 392-397. 